\title{
RELAÇÃO ENTRE O ESTADO NUTRICIONAL DE VITAMINA A E A REGRESSÃO DA ESTEATOSE HEPÁTICA APÓS GASTROPLASTIA EM Y- DE- ROUX PARA TRATAMENTO DA OBESIDADE CLASSE III
}

\author{
Relationship of the nutritional status of vitamin a and the regression of hepatic steatosis after \\ Roux-en-Y gastric bypass surgery for treatment of class III obesity
}

Luiz Gustavo de Oliveira e SILVA ${ }^{1}$, José Eduardo Ferreira MANSO ${ }^{1}$, Rejane Andréa Ramalho Nunes da SILVA ${ }^{2}$, Silvia Elaine PEREIRA ${ }^{2}$, Carlos José Saboya SOBRINHO ${ }^{3}$, Cesar Wakoff RANGEL ${ }^{1}$

Trabalho realizado no ${ }^{1}$ Programa de PósGraduação em Medicina (Cirurgia Geral)UFRJ, 2Instituto de Nutrição Josué de Castro - UFRJ e ${ }^{3}$ Clínica Cirúrgica Carlos Saboya. Rio de Janeiro, RJ, Brasil.

DESCRITORES - Estado nutricional. Vitamina A. Fígado gorduroso. Gastroplastia. Terapêutica. Obesidade mórbida.
RESUMO - Racional - A vitamina A participa de várias funções primordiais no organismo humano e as suas concentrações séricas podem estar diminuídas nas doenças crônicas não transmissíveis. Objetivo - Avaliar a relação entre o estado nutricional da vitamina $A$, e a regressão da esteatose hepática em indivíduos submetidos à gastroplastia em Y-de-Roux para tratamento da obesidade classe III. Métodos Foram estudados 30 pacientes obesos classe III, de ambos os sexos, com esteatose hepática, submetidos à gastroplastia em Y-de-Roux. Seis meses após a operação, os pacientes foram submetidos à ultrassonografia abdominal e distribuídos em dois grupos: grupo 1 - pacientes com esteatose detectada na ultrassonografia e grupo 2 - pacientes sem esteatose detectada na ultrassonografia. No pré-operatório e seis meses após a operação foram realizadas análises antropométricas e exames bioquímicos: insulina basal, glicemia, Homeostasis Model Assessment Index (HOMA IR), colesterol, HDL, LDL, triglicerídeos, AST, ALT, Gama-GT, albumina, bilirrubina total, retinol, e beta caroteno. Resultados - A média de perda de peso foi de 35,05 + 10,47 $(p<0,01)$ e a diminuição no Índice de Massa Corporal (IMC) de $11,6+4,99 \mathrm{Kg} / \mathrm{m}^{2}$ $(p<0,01)$. Após seis meses, todos os exames bioquímicos apresentaram diminuição significativa das concentrações $(p<0,05)$. O grupo 2 (sem esteatose) apresentou no pós-operatório, concentrações significativamente inferiores $(p<0,05)$, nas variáveis: peso, IMC, AST, ALT, gama GT, HOMA IR, insulina basal. As concentrações séricas de albumina apresentaram valores médios aproximados, sem diferença significativa nos dois tempos avaliados. Observaram-se concentrações séricas mais elevadas de retinol e de $\beta$-caroteno e redução menor em relação as concentrações do préoperatório nos pacientes sem esteatose, porém sem diferença estatística. Conclusão - Adequado estado nutricional de vitamina A pode contribuir na regressão da esteatose hepática em pacientes submetidos à Gastroplastia em Y-de-Roux para tratamento da obesidade classe III.

\section{Correspondência:}

José Eduado Ferreira Manso

jefmanso@osite.com.br

Fonte de financiamento: não há

Conflito de interesses: não há

Recebido para publicação: 21/05/2012 Aceito para publicação: 13/08/2012
ABSTRACT - Background - Vitamin A participates in several essentials functions in the human body and their serum concentrations may be decreased in non-transmissible diseases. Aim - To assess the relationship of the nutritional status of Vitamin A through the serum concentrations of retinol and beta carotene, with regression of hepatic steatosis in individuals who undergone Roux-en-Y gastric bypass surgery for treatment of class III obesity. Methods - Were included 30 individuals, male and female, submitted to Roux-en-Y gastric bypass for treatment of class III obesity, who were diagnosed through an abdominal ultrasonography as presenting hepatic steatosis. From the result of an ultrasonography screened six months after the surgical procedure those subjects were divided into two groups: group 1 patients with steatosis detected in the ultrasonography (16 subjects) and group 2 - patients without steatosis detected in the ultrasonography (14 subjects). Before and six months after the surgery, were carried out anthropometrical analyses and biochemical exams (basal insulin, glicemy, Homeostasis Model Assessment Index (HOMA IR), cholesterol, HDL, LDL, triglycerides, AST, ALT, Gamma-GT, albumin, total bilirubin, retinol, and beta carotene. Results - The individuals presented an average weight loss of $35.05+10.47(p<0.01)$ and a decrease in the Body Mass Index (BMI) of $11.6+4.99 \mathrm{Kg} / \mathrm{m}^{2}(\mathrm{p}<0.01)$. After six months, all the biochemical exams presented 
HEADINGS - Nutritional status. Vitamin A. Fatty liver. Gastroplasty. Therapeutics. Obesity, morbid a significant decrease in their basal concentrations $(p<0.05)$. In the post-operative period the group 2 (without steatosis) presented concentrations significantly lower $(p<0.05)$ in the following variables: weight, BMI, AST, ALT, Gamma-GT, HOMA IR, basal insulin. The albumin serum concentrations presented close average values, with no significant difference in the two periods evaluated. In the assessment of retinol and beta carotene, higher serum concentrations and a small decrease in relation to the concentrations of the pre-operative period in the group without steatosis were observed, however, no statistical difference was found. Conclusion - An adequate nutritional status of vitamin A might contribute in the improvement of the hepatic esteatosis after Roux-en-Y gastric bypass for class III obesity treatment.

\section{INTRODUÇÃO}

$\mathrm{O}$ indivíduo obeso apresenta alta incidência de diabete melito tipo 2, hipertensão arterial e dislipidemia, entre outras enfermidades ${ }^{24}$. A doença hepática gordurosa não-alcoólica (DHGNA), frequentemente associada à obesidade, apresenta espectro amplo de agressão hepática que pode variar da esteatose à esteatohepatite, fibrose avançada e cirrose ${ }^{1}$. Esta doença ocorre em $3 \%$ a $24 \%$ da população geral e é muito comum entre os pacientes que são submetidos à cirurgia bariátrica, variando de $84 \%$ a $96 \% 5$.

A vitamina A participa de várias funções primordiais no organismo humano, tais como: acuidade visual, atividade imunológica, proliferação e diferenciação celular ${ }^{12}$. Apesar de gestantes, nutrizes, recém-nascidos, lactentes e préescolares constituírem os grupos clássicos mais vulneráveis para a hipovitaminose $A$, a literatura aponta concentrações séricas de vitamina A diminuídas em indivíduos com doenças que envolvam a absorção de lipídios e também em distúrbios metabólicos, como doenças do fígado e no diabete melito tipo $2^{9,28}$. Recentemente, esta vitamina, assim como os seus precursores, receberam destaque pela sua atuação contra os radicais livres, protegendo o organismo contra o estresse oxidativo e, consequentemente, prevenindo danos e lesões teciduais relacionados às diversas doenças crônicas não transmissíveis?

A deficiência de vitamina A pode estar presente em indivíduos com DHGNA, contribuindo para evolução da doença, pois estes indivíduos estão mais susceptíveis ao estresse oxidativo que, por si só, aumenta em muito o consumo de substâncias com função antioxidante ${ }^{27}$.

Tendo em vista os fatos descritos acima e a eficiência do tratamento operatório da obesidade classe III na diminuição das complicações desta doença ${ }^{3,6}$, incluindo a esteatose hepática ${ }^{23}$, foi proposto avaliar a relação entre o estado nutricional da vitamina $A$, através das concentrações séricas de retinol e $\beta$-caroteno, e a regressão da esteatose hepática nos pacientes submetidos à gastroplastia em Y-de-Roux (GYR) para o tratamento da obesidade classe III.

\section{MÉTODOS}

Este trabalho foi aprovado pelo Comitê de Ética e Pesquisa do Hospital Universitário Clementino Fraga
Filho (CAE 007.0.197197-06).

Foram avaliados 30 indivíduos obesos de ambos os sexos com diagnóstico de esteatose hepática submetidos à GYR para tratamento da obesidade classe III. Os critérios de inclusão foram: indicação cirúrgica para tratamento da obesidade (IMC $>40 \mathrm{Kg} / \mathrm{m}^{2}$ ou IMC $>35 \mathrm{Kg} / \mathrm{m}^{2}$ com co-morbidades); diagnóstico de esteatose hepática realizado através da ultrassonografia abdominal no pré-operatório; realização de avaliações antropométricas pela equipe médica antes e seis meses após a operação; realização de exames bioquímicos e de ultrassonografia antes e seis meses após a operação.

A inclusão de cada paciente no estudo foi feita mediante autorização formal, através da assinatura de termo de consentimento, após esclarecimentos sobre os objetivos e procedimentos do projeto por parte do pesquisador, de acordo com as normas estabelecidas pelo Conselho Nacional de Saúde (1987). Os pacientes foram informados que, em contrapartida à sua participação no estudo, teriam diagnóstico nutricional, tratamento das carências nutricionais diagnosticadas, educação nutricional individualizada e que os resultados obtidos lhes seriam informados. Todos os pacientes foram orientados a utilizar polivitamínicos com dosagem diária de 5000 UI de acetato de retinol, após a operação. A equipe cirúrgica foi a mesma em todos os procedimentos.

Os critérios de exclusão foram: recusa em participar do estudo; presença de síndromes disabsortivas, infecções agudas e crônicas; gestantes e nutrizes; endocrinopatias associadas; consumo de álcool superior a $20 \mathrm{~g} /$ dia; doença alcoólica do fígado, hepatite viral, hepatite auto-imune, cirrose biliar primária, colangite esclerosante primária, doenças metabólicas do fígado e hepatite induzida por drogas ou qualquer outra doença hepática que não seja a DHGNA; ter feito operação bariátrica prévia.

Foram analisadas as seguintes variáveis: peso, estatura e índice de massa corporal (IMC); resistência insulínica, determinada pelo Homeostasis Model Assessment Index $(\mathrm{HOMA})^{20}$, concentração plasmática de insulina basal, expressa em $\mathrm{mcu} / \mathrm{ml}$; concentrações plasmáticas de glicose de jejum, expressa em mg/ dl; perfil lipídico, avaliado através da dosagem sérica da concentração de colesterol total, triglicerídeos, lipoproteína de alta densidade (HDL) e lipoproteína de baixa densidade (LDL), expressos em $\mathrm{mg} / \mathrm{dL}$; provas 
de função e lesão hepática, através dosagem sérica da concentração de albumina $(\mathrm{g} / \mathrm{dL})$, bilirrubina total (g/dL), e gama-glutamiltranspeptidase (GGT) (U/L), e AST (U/L), ALT (U/L); estado nutricional da vitamina $A$, através da determinação das concentrações séricas de retinol e $\beta$-caroteno, pelo método HPLC (High Performance Liquid Chromatography), expressas em $\mu \mathrm{mol} / \mathrm{L}$ e $\mu \mathrm{g} / \mathrm{dL}$, respectivamente.

O procedimento cirúrgico realizado nestes pacientes foi a GYR que consiste na confecção de um pequeno reservatório gástrico vertical, com reconstrução do trânsito em $\mathrm{Y}$-de-Roux. A anastomose gastrojejunal era manual e calibrada $(0,8-1,2 \mathrm{~cm})$ e a anastomose jejunojejunal feita a $100-150 \mathrm{~cm}$ da anterior. A operação foi realizada pelo método convencional ou videolaparoscópico ${ }^{13}$.

Foram realizadas duas análises: uma avaliação geral, constatando o efeito da operação no grupo total (30 pacientes), comparando as variáveis do pósoperatório (seis meses) com as do pré-operatório.

A outra análise foi feita a partir da distribuição dos indivíduos em dois grupos: grupo 1 - pacientes com esteatose, grupo 2 - pacientes sem esteatose, a partir da ultrassonografia realizada no pós-operatório. Desta forma, procurou-se identificar se havia alguma influência do estado nutricional de vitamina A nos pacientes que apresentavam regressão do grau de doença hepática.

Para análise dos resultados foram utilizados testes não paramétricos (Wilcoxon e Mann-Whitney) para as variáveis que não apresentavam distribuição normal. Para aquelas que a apresentavam foi utilizado o teste t-Student, $\mathrm{O}$ teste da normalidade foi realizado através do teste de Kolmogorov-Smirnov. O nível de significância estabelecido foi de 0,05.

\section{RESULTADOS}

\section{Avaliação geral}

Entre os indivíduos estudados, 19 (63,3\%) eram do sexo feminino e 11 (36,7\%), do sexo masculino. A média de idade do grupo foi de $43,15 \pm 11,36$ anos, variando de 19 a 60 anos. Excetuando-se o HDL e a albumina, todas as variáveis estudadas apresentaram redução significativa $(p<0,05)$, seis meses após a operação (Tabela 1).

Após a realização da ultrassonografia no pósoperatório identificou-se que 16 indivíduos (53,3\%) mantinham esteatose hepática e 14 (46,6\%) não.

\section{Avaliação entre os grupos}

\section{Pós-operatório}

A partir dos resultados obtidos em cada grupo, seis meses após a operação, observou-se que 11 pacientes sem esteatose hepática eram do sexo feminino e três do masculino. Entre os indivíduos do grupo 1 (com esteatose), havia oito homens e oito mulheres. A
TABELA 1 - Variáveis estudadas antes e seis meses após GYR (avaliação geral)

\begin{tabular}{|c|c|c|c|}
\hline & Pré-operatório & $\begin{array}{l}\text { Pós-operatório } \\
\text { (seis meses) }\end{array}$ & $p$ \\
\hline Peso (Kg) & $129,9+23,1$ & $94,95+24,46$ & $<0,01^{*}$ \\
\hline $\operatorname{IMC}\left(\mathrm{Kg} / \mathrm{m}^{2}\right)$ & $45,9+5.63$ & $34,31+8.17$ & $<0,01^{*}$ \\
\hline Glicose (mg/dL) & $114,4+37,3$ & $89,6+13.1$ & $0,002^{* *}$ \\
\hline HOMA IR & $5.95+2.78$ & $2,44+1,57$ & $<0,01^{* *}$ \\
\hline Insulina $(\mu \mathrm{U} / \mathrm{mL})$ & $23,67+10,9$ & $9,74+5,46$ & $<0,01^{* *}$ \\
\hline Colesterol (mg/dL) & $195+35,81$ & $162,53+31,93$ & $<0,01^{*}$ \\
\hline $\mathrm{HDL}(\mathrm{mg} / \mathrm{dL})$ & $46,31+10,6$ & $43,6+9,72$ & $0,182^{*}$ \\
\hline $\mathrm{LDL}(\mathrm{mg} / \mathrm{dL})$ & $113,41+27,6$ & $92,66+23,29$ & $<0,01^{*}$ \\
\hline Triglicerídeos (mg/dl) & $175,63+10,34$ & $111,33+34,92$ & $<0,01^{* *}$ \\
\hline AST (U/L) & $32,58+13,24$ & $23,1+10,39$ & $0,01^{* *}$ \\
\hline ALT (U/L) & $45,17+20$ & $24,2+14,8$ & $<0,01^{* *}$ \\
\hline GGT (U/L) & $44,11+23,89$ & $18,7+14,9$ & $<0,01^{* *}$ \\
\hline BT (mg/dL) & $0,61 \pm 0,14$ & $0,8 \pm 0,18$ & $<0,01^{* *}$ \\
\hline Albumina (g/dL) & $4,02+0,43$ & $4,18+0,33$ & $0,09 * *$ \\
\hline Retinol $(\mu \mathrm{mol} / \mathrm{L})$ & $1,69+0,55$ & $1,34+0,57$ & 0,019 * \\
\hline B-caroteno $(\mu \mathrm{g} / \mathrm{dl})$ & $68,84+48$ & $33,8+23,39$ & $<0,01^{* *}$ \\
\hline \multicolumn{2}{|c|}{$\begin{array}{c}\text { Negrito }=\text { resultado significativo } \\
B T=\text { bilirrubina total }\end{array}$} & \multicolumn{2}{|c|}{$\begin{array}{l}\text { * - Teste t } \\
\text { ** - Teste Wilcoxon }\end{array}$} \\
\hline
\end{tabular}

análise da média de idade dos grupos mostrou que os pacientes sem esteatose hepática $(40,29 \pm 11,77)$ eram mais jovens do que os pacientes com ela $(46,44 \pm 10,51)$.

O grupo 2 apresentou diminuição significativa do peso $(p=0,048)$, IMC $(p=0,01)$, RI (HOMA IR- $p=0,049)$, insulina basal $(p=0,02)$ e enzimas hepáticas (AST $p=0,012 ;$ ALT $p=0,02 ;$ GGT $p=0,01$ ) (Tabela 2).

TABELA 2 - Comparação entre os grupos 1 e 2 seis meses após a operação

\begin{tabular}{|c|c|c|c|}
\hline & $\begin{array}{c}\text { GRUPO } 1 \\
\text { COM ESTEATOSE }\end{array}$ & $\begin{array}{c}\text { GRUPO } 2 \\
\text { SEM ESTEATOSE }\end{array}$ & $\mathrm{p}$ \\
\hline Peso $(\mathrm{Kg})$ & $103,91+26,17$ & $84,71+18,26$ & $0,048^{*}$ \\
\hline $\operatorname{IMC}\left(\mathrm{Kg} / \mathrm{m}^{2}\right)$ & $37,85+8,25$ & $30,28+6.14$ & $0,011^{*}$ \\
\hline Glicose (mg/dL) & $89,25+10,24$ & $90+16.06$ & $0,917^{\star}$ \\
\hline HOMA IR & $2.97+1,86$ & $1,85+0,89$ & 0,049 ** \\
\hline Insulina $(\mu \mathrm{U} / \mathrm{mL})$ & $11,8+6,25$ & $7,41+6,23$ & $0,022^{* *}$ \\
\hline Colesterol (mg/dL) & $162,25+28,6$ & $162,86+36,48$ & $0,96^{*}$ \\
\hline $\mathrm{HDL}(\mathrm{mg} / \mathrm{dL})$ & $41,56+8,63$ & $45,93+10,69$ & $0,226^{*}$ \\
\hline $\mathrm{LDL}(\mathrm{mg} / \mathrm{dL})$ & $97,31+18,82$ & $87,36+27,29$ & $0,25^{*}$ \\
\hline Triglicerídeos (mg/dl) & $111,19+27,67$ & $111,50+42,87$ & $0,982^{*}$ \\
\hline AST (U/L) & $26,81+10,89$ & $18,86+8,23$ & $0,012^{* *}$ \\
\hline ALT (U/L) & $29,94+15,89$ & $17,64+10,67$ & 0,02 ** \\
\hline GGT (U/L) & $25,56+17,49$ & $10,86+4,28$ & $0,01^{* *}$ \\
\hline BT (mg/dL) & $0,69 \pm 0,18$ & $0,65 \pm 0,19$ & $0,692^{* *}$ \\
\hline Albumina (g/dL) & $4,21+0,35$ & $4,15+0,31$ & $0,651^{*}$ \\
\hline \multicolumn{2}{|c|}{$\begin{array}{c}\text { Negrito }=\text { resultado significativo } \\
B T=\text { bilirrubina total }\end{array}$} & \multicolumn{2}{|c|}{$\begin{array}{l}\text { * - Teste } t \\
\text { ** - Teste Wilcoxon }\end{array}$} \\
\hline
\end{tabular}

A análise do perfil lipídico no pós-operatório não evidenciou diferença significante entre os dois grupos. Da mesma forma, a glicemia e a albuminemia apresentaram-se bastante semelhantes (Tabela 2).

\section{Variação entre pré e pós-operatório}

Observou-se redução em relação aos resultados do pré-operatório mais acentuada no grupo 2 nas variáveis peso, IMC, AST, ALT, colesterol, LDL, 
triglicerídeos - porém, sem significância estatística. (Tabela 3).

TABELA 3 - Comparação da variação entre pré e pósoperatório nos grupos 1 e 2

\begin{tabular}{|c|c|c|c|}
\hline & $\begin{array}{c}\text { GRUPO } 1 \\
\text { COM ESTEATOSE }\end{array}$ & $\begin{array}{c}\text { GRUPO } 2 \\
\text { SEM ESTEATOSE }\end{array}$ & $p$ \\
\hline Peso $(\mathrm{Kg})$ & $33,71 \pm 11,41$ & $37,03 \pm 9,29$ & $0,29 *$ \\
\hline $\operatorname{IMC}\left(\mathrm{Kg} / \mathrm{m}^{2}\right)$ & $9,9 \pm 4,69$ & $13,53 \pm 4,77$ & $0,06^{*}$ \\
\hline Glicose (mg/dL) & $28,13 \pm 38,61$ & 21,00 & $0,636^{* *}$ \\
\hline HOMA IR & $3,77 \pm 3,01$ & $3,43 \pm 3,03$ & $0,774 *$ \\
\hline Insulina $(\mu \mathrm{U} / \mathrm{mL})$ & $15,85 \pm 10,81$ & $11,72 \pm 7,92$ & $0,262^{* *}$ \\
\hline Colesterol (mg/dL) & $28,4 \pm 34,61$ & $36,36 \pm 25,87$ & $0,492^{*}$ \\
\hline $\mathrm{HDL}(\mathrm{mg} / \mathrm{dL})$ & $3,33 \pm 7,51$ & $1,14 \pm 9,24$ & $0,488^{*}$ \\
\hline LDL (mg/dL) & $13,33 \pm 24,4$ & $29 \pm 23,4$ & $0,087^{*}$ \\
\hline Triglicerídeos (mg/dl) & $49,88 \pm 48,79$ & $80,79 \pm 106,78$ & $0,306^{*}$ \\
\hline AST (U/L) & $8,2 \pm 11,29$ & $12,36 \pm 16,96$ & $0,431^{*}$ \\
\hline ALT (U/L) & $19,87 \pm 21,07$ & $24,43 \pm 24,11$ & $0,591^{*}$ \\
\hline GGT (U/L) & $27,78 \pm 32,14$ & $26,54 \pm 18,69$ & $0,847^{*}$ \\
\hline BT (mg/dL) & $0,22 \pm 0,21$ & $0,13 \pm 0,25$ & $0,618^{* *}$ \\
\hline Albumina (g/dL) & $-0,23 \pm 0,44$ & $-0,04 \pm 0,41$ & $0,223^{*}$ \\
\hline \multicolumn{2}{|c|}{$\begin{array}{c}\text { Negrito }=\text { resultado significativo } \\
\qquad \mathrm{BT}=\text { bilirrubina total }\end{array}$} & \multicolumn{2}{|c|}{$\begin{array}{l}\text { * }- \text { Teste } t \\
{ }^{* *} \text { - Teste Wilcoxon }\end{array}$} \\
\hline
\end{tabular}

\section{Concentrações de retinol e $\beta$-caroteno}

Observou-se que a média das concentrações séricas de retinol, no pós-operatório, apresentou-se mais elevada no grupo $2(1,50+0,56 \mu \mathrm{mol} / \mathrm{l})$ em relação ao grupo $1(1,21+0,57 \mu \mathrm{mol} / \mathrm{l})$, apesar de não ter sido encontrado significância estatística $(p=0,171)$. Da mesma forma, observou-se redução menos acentuada do retinol nestes indivíduos, a partir dos resultados do pré-operatório $(p=0,398)$ (Figura 1$)$.

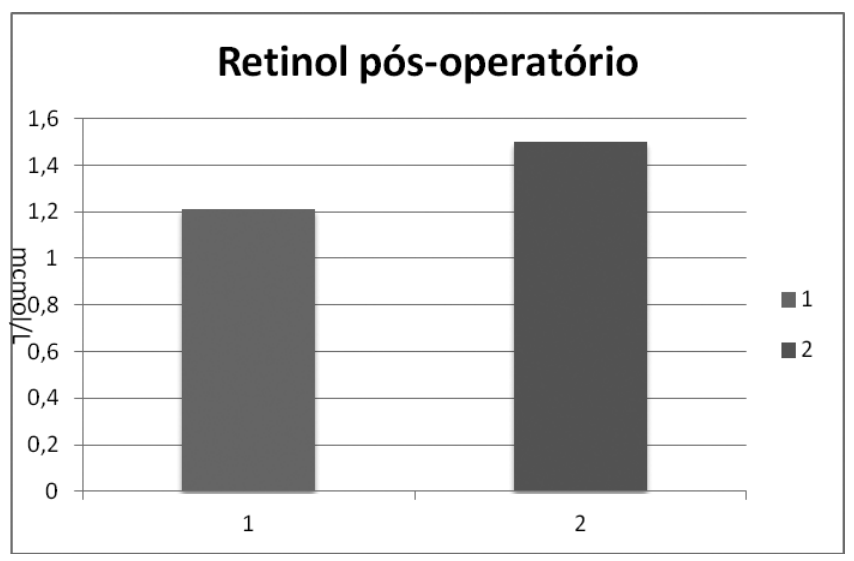

FIGURA 1 - Concentrações séricas de retinol seis meses após a operação nos grupos 1 e 2. $p=0,171$ (Teste $t$ )

O comportamento do $\beta$-caroteno seis meses após a operação foi semelhante ao do retinol, apresentando médias séricas mais elevadas e variação entre pré e pós-operatório menos acentuada no grupo 2. Estes resultados não foram considerados significantes (Figuras 2 e 3).

\section{Variação Retinol}

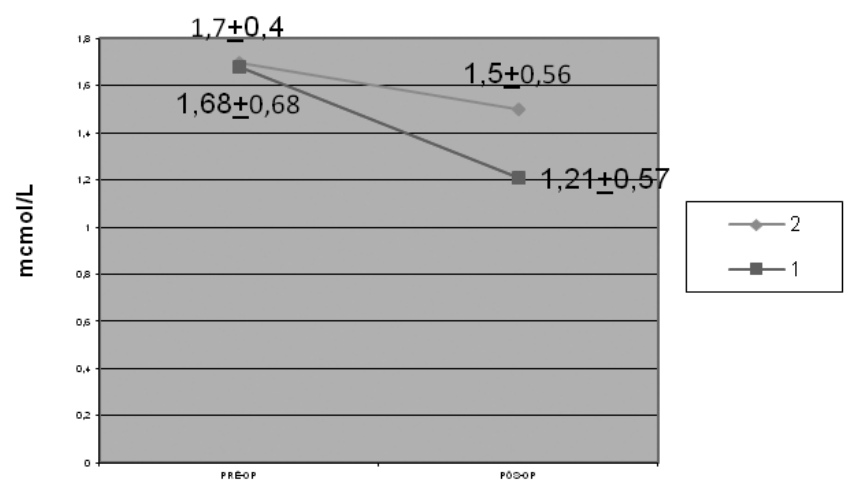

FIGURA 2 - Variação das concentrações séricas de retinol entre o pré e pós-operatório. $p=0,398$ (Teste $t$ ).

\section{Betacaroteno pós-operatório}

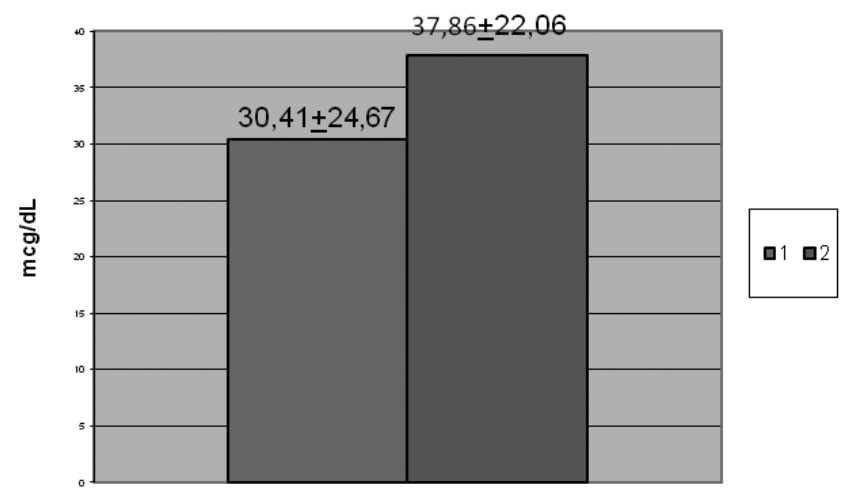

FIGURA 3 - Concentrações séricas de $\beta$-caroteno seis meses após a operação nos grupos 1 e 2. $p=0,219$. (Teste Mann Whitney)

\section{DISCUSSÃO}

\section{Avaliação geral}

O tratamento cirúrgico é o mais efetivo para pacientes portadores de obesidade classe $\mathrm{III}^{6}$. Neste estudo foi encontrada média de perda de peso de 35,05 $\pm 10,47 \mathrm{Kg}(26,98 \%)$ e diminuição de IMC de $11,6 \pm$ $4,99 \mathrm{Kg} / \mathrm{m}^{2}$ nos seis primeiros meses após a operação. Este resultado pode ser considerado satisfatório, visto que houve melhora significativa em vários parâmetros metabólicos, como a RI, o perfil lipídico e as enzimas hepáticas.

Buchwald et al. ${ }^{14}$, observaram, após a GYR, resolução do diabete melito tipo 2 em $78,1 \%$ dos pacientes e resolução e melhora em $86,6 \%$. Estes autores também detectaram em relação à dislipidemia, avaliando vários tipos de operações, redução de $33,20 \mathrm{mg} / \mathrm{dL}$ nas concentrações séricas de colesterol total, $29,34 \mathrm{mg} / \mathrm{dL}$ nas de LDL e 79,65 mg/dL nas de triglicerídeos ${ }^{15}$. Estes resultados foram semelhantes aos encontrados neste estudo onde houve diminuição de 32,$24 ; 20,79$ e $64,30 \mathrm{mg} / \mathrm{dL}$, respectivamente, 
nas concentrações séricas de colesterol total, LDL e triglicerídeos $(p<0,01)$.

$O$ efeito benéfico da cirurgia bariátrica sobre a DHGNA, através da redução do peso e da melhora da síndrome metabólica, já foi relatado ${ }^{16,17,18}$. Mattar et al. acompanharam 70 pacientes submetidos à cirurgia bariátrica, com diagnóstico de DHGNA confirmado por biópsia hepática e encontraram resolução da esteatose hepática em $37 \%$ dos pacientes e da fibrose, em $20 \%$. Houve redução significativa no peso, IMC, glicemia, colesterol total, triglicerídeos, LDL, AST e ALT ${ }^{19}$.

Nesta análise, a esteatose hepática não foi detectada na ultrassonografia em $46,6 \%$ dos indivíduos estudados seis meses após a operação, mas para confirmação do desaparecimento da esteatose, haveria necessidade da realização de biópsia hepática. Entretanto, foi observada melhora significativa de vários parâmetros metabólicos considerados fatores de risco para a DHGNA. Este fato pode ter contribuído para a regressão da esteatose hepática nestes pacientes.

\section{Comparação entre os grupos 1 e 2}

A partir dos resultados obtidos no pós-operatório, observou-se diferença no perfil dos grupos. Os pacientes nos quais a ultrassonografia não identificou esteatose hepática (grupo 2) apresentavam peso e IMC menores, concentrações séricas de HOMA-IR, insulina basal e enzimas hepáticas significativamente menores. Com isto, pôde-se distinguir este grupo como de menor peso, menor grau de RI e com marcadores séricos de lesão hepática mais reduzidos. Estes dados evidenciaram as características do paciente com DHGNA. Marchesini et al. ${ }^{20}$ provaram, utilizando o HOMA, que a resistência insulínica era o achado laboratorial mais diretamente relacionado com a DHGNA. A forte associação desta doença com obesidade, distribuição gordurosa central, diabete melito tipo 2, dislipidemia, hipertensão e doença aterosclerótica, suporta a hipótese que a DHGNA possa representar mais uma condição da síndrome metabólica ${ }^{21}$.

Em estudo realizado em 1022 coreanos com intenção de correlacionar síndrome metabólica com a gravidade da esteatose hepática (graduada através de critérios ultrassonográficos), foi encontrado que à medida que o grau aumentava, havia incremento no peso, na RI (aferida através do HOMA), nas concentrações séricas de enzimas hepáticas e também nas de colesterol e triglicerídeos ${ }^{22}$.

Segundo alguns estudos que avaliaram a epidemiologia da DHGNA, referem ser mais comum em homens e a sua incidência cresce com o aumento da faixa etária ${ }^{3,23}$. Este fato está de acordo com o achado da nesta série onde a predominância do sexo feminino foi maior no grupo 2 (11/14). Já entre os indivíduos portadores de esteatose hepática, esta relação diminuiu, pois foram encontrados homens e mulheres na mesma proporção. Em relação à idade, os pacientes sem ela apresentaram média de idade mais baixa $(40,29 \pm 11,77)$ em relação aos com $(46,44 \pm 10,51)$; entretanto, não houve significância estatística nesta análise $(p>0,05)$.

Quando foi observada a variação entre pré e pósoperatório, pôde-se constatar que a operação provocou alterações muito semelhantes nos dois grupos. Desta forma, fica evidente que a GYR foi eficaz para todos os indivíduos, sendo que alguns, provavelmente por apresentarem fatores de risco para a DHGNA como obesidade, RI, dislipidemia e estresse oxidativo, mais intensos no pré-operatório, ainda mantiveram grau de doença hepática um pouco mais avançado no momento em que foi feita a análise.

\section{Avaliação do estado nutricional de vitamina $A$ após a operação \\ Após a GYR observou-se diminuição significativa} nas concentrações de retinol e $\beta$-caroteno. Isto pode ser justificado porque a vitamina A é lipossolúvel 24 e após a operação a absorção de lipídeos é menor, devido à ingestão reduzida e ao bypass jejunal. Além disto, a ingestão de alimentos fonte de vitamina A, em geral, também está reduzida. A deficiência de vitamina A após a GYR já foi relatada anteriormente 25 e há casos descritos de xeroftalmia e cegueira noturna ${ }^{26}$.

Foi constatado neste estudo que a diminuição das concentrações séricas de $\beta$-caroteno após a operação, foi maior em relação às de retinol. Sabe-se que o $\beta$-caroteno é o mais potente precursor do retinol, podendo ser convertido, conforme à biodisponibilidade do retinol ${ }^{12}$. Portanto, esta maior redução das concentrações de $\beta$-caroteno pode ser justificada pela conversão deste à retinol. Os indivíduos do grupo 1 apresentavam concentrações séricas de retinol e $\beta$-caroteno menores. Apesar de não ter apresentado significância estatística, este fato sugere que a vitamina A pode estar relacionada com a regressão da esteatose hepática após a gastroplastia em Y-de-Roux.

O depósito excessivo de ácidos graxos no fígado promove aumento no estresse oxidativo, o qual pode ser responsável pela progressão de esteatose hepática para esteatose hepática não alcoólica e posteriormente para cirrose ${ }^{2}$. O uso de antioxidantes, como as vitaminas E e C no tratamento da DHGNA, apresenta resultados satisfatórios, com regressão da inflamação e fibrose ${ }^{27}$.

O retinol e os carotenóides são eficientes varredores de radicais livres, que protegem o organismo contra o estresse oxidativo e, consequentemente, da lesão celular. Portanto, como o paciente com DHGNA possui concentrações elevadas de peroxidação lipídica, eles podem apresentar aumento da utilização de substâncias antioxidantes, entre elas a vitamina $A$.

Após o tratamento cirúrgico, em decorrência da perda de peso e da melhora das co-morbidades há diminuição do estresse oxidativo. A peroxidação lipídica, avaliada pelas concentrações plasmáticas de malondialdeído, diminui significativamente 12 e 24 semanas após a gastroplastia vertical com bandagem 
e as concentrações séricas de $\alpha$-tocoferol aumentam significativamente 24 semanas após a operação, já as concentrações de $\beta$-caroteno apresentam aumento não significativo ${ }^{28}$. Portanto, espera-se que, após a GYR, ocorra diminuição da peroxidação lipídica, com consequente melhora da DHGNA e aumento das concentrações de substâncias antioxidantes.

Apesar das concentrações séricas médias de retinol e $\beta$-caroteno apresentarem-se reduzidas nos pacientes que mantinham esteatose na ultrassonografia após a operação, não houve comprovação desta relação através da análise estatística empregada na presente análise. Justificativa para este resultado pode ser o pequeno número de indivíduos estudados (30 pacientes). Talvez, em estudo utilizando amostra maior, esta relação possa tornar-se significativa. Os relatos na literatura da relação entre as concentrações séricas de vitamina $A$ e DHGNA são escassos. Em estudo utilizando modelo experimental de ratos transgênicos com receptores hepáticos de ácido retinóico defeituosos, observou-se desenvolvimento de esteatose hepática não alcoólica aos quatro meses de idade e hepatocarcinoma aos 12 . Os animais que receberam dieta rica em ácido retinóico desde três semanas de vida não desenvolveram alterações histológicas no fígado. Estes fatos sugerem efeito protetor do ácido retinóico no desenvolvimento de DHGNA e hepatocarcinoma ${ }^{29}$.

Rocchi et al., avaliaram o nível de vitaminas antioxidantes lipossolúveis em pacientes com cirrose hepática comparado a controles sadios e observaram concentrações significativamente reduzidas de retinol e demais vitaminas lipossolúveis nesses pacientes ${ }^{30}$.

Yadav et al. observaram que as concentrações séricas de retinol e demais antioxidantes estavam acentuadamente depletadas em pacientes com doença hepática e que o agravo da fibrose foi associado com o decréscimo do retinol e dos demais antioxidantes no fígado, podendo ser consequência da depleção de antioxidantes ou do decréscimo de estoque hepático em decorrência da fibrose ${ }^{31}$.

Estes achados são diferentes daqueles encontrados no neste estudo, onde o objetivo era detectar se o efeito antioxidante da vitamina A apresentar-seia evidente com a regressão da esteatose hepática ocorrida após o tratamento cirúrgico. Apesar de não ter sido comprovado estatisticamente, observou-se que a diminuição das concentrações séricas de retinol em relação ao pré-operatório foi menor nos pacientes onde se identificou maior regressão da esteatose hepática. Quando é analisada a mesma variação em relação ao $\beta$-caroteno, identifica-se redução bem menos acentuada nestes pacientes. Isto é importante, visto que o $\beta$-caroteno possui efeito antioxidante mais intenso em relação ao retinol e pode ter facilitado a regressão da esteatose hepática neste grupo de pacientes.

Como discutido anteriormente, vários estudos evidenciam a relação da gravidade da doença hepática com baixas concentrações séricas de vitamina $A$. Entretanto, as publicações relacionando vitamina A com DHGNA ainda são escassas e conflitantes, caracterizando esta relação como um campo aberto para novas pesquisas.

\section{CONCLUSÃO}

Houve distinção entre os dois grupos analisados. Os pacientes que não mantinham sinais de esteatose hepática na ultrassonografia seis meses após o procedimento, apresentavam alterações metabólicas compatíveis com um nível menor de estresse oxidativo, concentrações séricas maiores de retinol e $\beta$-caroteno no pós-operatório, e redução menor destas concentrações em relação ao pré-operatório. Apesar de não ter comprovação estatística, os indivíduos que mantinham esteatose hepática provavelmente tiveram consumo maior de substâncias antioxidantes, como o retinol e $\beta$-caroteno. Pode-se inferir que bom estado nutricional de vitamina A contribui na regressão da esteatose hepática em pacientes submetidos à gastroplastia em Y-de-Roux para tratamento da obesidade classe III.

\section{REFERÊNCIAS}

1. Angulo P. Nonalcoholic Fatty Liver Disease. N Engl J Med. 2002;346(16):1221-31.

2. Blomhoff R. Transport and metabolism of vitamin A. Nutr Rev. 1994;52(2 Pt 2):S13-23.

3. Branco-Filho AJ, Menacho AM, Nassif LS, Hirata LM, Gobbi RIS, Perfete C, Siqueira DED. Gastroplastia como tratamento do diabete melito tipo 2. ABCD, arq. bras. cir. dig. 2011;24(4):285-9.

4. Buchwald H, Estok R, Fahrbach $K$, Banel D, Jensen MD, Pories WJ, Bantle JP, Sledge I. Wheight and type 2 diabetes after bariatric surgery: systematic review and meta-analysis.Am J Med. 2009;122(3):248-56.

5. Clark JM. The epidemiology of nonalcoholic fatty liver disease in adults. J Clin Gastroenterol. 2006; 40(3 Supl.1):S5-10.

6. Costa LD,Valezi AC, Matsuo T, Dichi I, Dichi JB. Repercussão da perda de peso sobre parâmetros nutricionais e metabólicos de pacientes obesos graves após um ano de gastroplastia em Y-deRoux. Rev Col Bras Cir. 2010;37(2):96-101.

7. Delport R, Ubbink JB, Human JA, Becker PJ, Myburgh DP, Vermaak WJ. Antioxidant vitamins and coronary artery disease risk in South African males. Clin Chim Acta. 1998;278(1):55-60.

8. Eckert MJ, Perry JT, Sohn VY. Incidence of low vitamin A levels and ocular symptoms after Roux-en-Y gastric bypass. Surg Obes Relat Dis. 2010;6(6):653-7.

9. Gerster $\mathrm{H}$. Vitamin A - functions, dietary requirements and safety in humans. Internat. J. Vit. Nutr. Res. 1997;67:71-90.

10. Harrison SA, Torgerson S, Hayashi P, Ward J, Schenker S. Vitamin $E$ and vitamin $C$ treatment improves fibrosis in patients with nonalcoholic steatohepatites. Am J Gastroenterology. 2003;98(11):2485-90.

11. Henry Buchwald, MD, Yoav Avidor, Eugene Braunwald, Michael D. Jensen, Walter Pories, Kyle Fahrbach, Karen Schoelles, Henry Buchwald, Yoav Avidor, Eugene Braunwald, Michael D. Jensen, Walter Pories, Kyle Fahrbach, Karen Schoelles. Bariatric surgery. A sistematic review and meta-analysis. JAMA. 2004;292(14):1724-37.

12. Institute of Medicine (IOM). Vitamin A. IN: Dietary reference intakes for vitamin A, vitamin K, arsenic, boron, chromium, copper, iodine, iron, manganese, molybdenum, nickel, silicon, vanadium, and zinc. Washington: National Academy Press; 2001. p. 82-161 
13. KD Higa, KB Boone, T Ho, OG Davies. Laparoscopic Roux-en-Y Bypass for Morbid Obesity. Technique and preliminary results of our first 400 patients. Arch Surg. 2000;135:1029-34.

14. Kim HC, Choi SH, Shin HW, Cheong JY, Lee KW, Lee HC, Huh KB, Kim DJ. Severity of ultrasonographic liver steatosis and metabolic syndrome in Korean men and women. World J Gastroenterol. 2005;11(34):5314-21.

15. Kisakol G, Guney E, Bayraktar F, Yilmaz C, Kabalak T, Ozmen D. Effect of surgical weight loss on free radical and antioxidant balance: a preliminary report. Obes Surg. 2002;12(6):795-801.

16. Klein S, Mittendorfer B, Eagon JC, Patterson B, Grant L, Feirt N, Seki E, Brenner D, Korenblat K, McCrea J. Gastric Bypass Surgery improves metabolic and Hepatic Abnormalities associated with Nonalcoholic Fatty Liver Disease. Gastroenterology. 2006;130:1564-72.

17. Madan AK, Orth WS, Tichansky DS, Ternovits CA. Vitamin and trace material level after laparoscopic gastric bypass. Obes Surg, 2006;16(5):603-6.

18. Marchesini G, Brizi M, Morselli-Labate AM, Bianchi G, Bugianesi E, McCullough AJ, Forlani G, Melchionda N. Association of nonalcoholic fatty liver disease to insulin resistance. Am J Med. 1999;107(5:450-5.

19. Mattar SG, Velcu LM, Rabinovitz M, Demetris AJ, Krasinskas AM, Barinas-Mitchell E, Eid GM, Ramanathan R, Taylor DS, Schauer PR.. Surgically-induced weight loss significantly improves nonalcoholic fatty liver disease and the metabolic syndrome. Ann Surg. 2005;242(4):610-20.

20. Matthews DR, Hosker JP, Rudenski AS, Naylor BA, Treacher DF, Turner RC. Homeostasis model assessment: insulin resistance and beta-cell function from fasting plasma glucose and insulin concentration in man. Diabetologia. 1985;28(7):412-9.

21. Mummadi RR, Kasturi K.S, Chennareddygari S. Effect of bariatric surgery on nonalcoholic fatty liver disease: systematic review and meta-analysis. Clin Gastroenterol Hepatol. 2008;6(12):1396-402.
22. Paredes $A H$, Torres DM, Harrison SA. Non Alcoholic Fatty Liver Disease. Clin Liver Dis. 2012;16(2):397-419.

23. Pillai AA, Rinella ME. NAFLD, Non-alcoholic fatty liver disease: is bariatric surgery the answer? Clin Liver Dis. 2009;13(4):689-710.

24. Plecka Östlund M. et al. Morbidity and mortality before and after bariatric surgery for morbid obesity compared with the general population. Br J Surg. 2011; 98(6):811-6.

25. Rocchi E, Borghi A, Paolillo F, Pradelli M, Casalgrandi G. Carotenoids and lipossoluble vitamins in liver cirrhosis. J Lab Clin Med. 1991;118:176-85.

26. Ruhl CE, Everhart JE. Determinants of the association with overweight with elevated serum alanine aminotrasferase activity in United States. Gastroenterology. 2003;124(1):71-9.

27. Sarni RO Ramalho RA. Serum retinol and total carotene concentrations in obese children. Med. Sci. Monit. 2005; 11(11):CR510-514

28. Underwood BA, Arthur A. The contribuition of vitamin A to public health. Faseb J. 1996;10:1040-8.

29. Weiner RA. Surgical treatment of non-alcoholic steatohepatitis and non-alcoholic fatty liver disease. Dig Dis. 2010;28(1):274-9.

30. Yadav D, Hertan HI, Schweitzer P, Norkus EP, Pitchumoni CS. Serum and liver micronutrient antioxidants and serum oxidative stress in patients with cronic hepatitis C. Am J Gastroenterol. 2002;97(10):2634-9.

31. Yanagitani A, Yamada S, Yasui S, Shimomura T, Murai R, Murawaki $Y$, Hashiguchi K, Kanbe T, Saeki T, Ichiba M, Tanabe Y, Yoshida $Y$, Morino S, Kurimasa A, Usuda N, Yamazaki $H$, Kunisada $T$, Ito H, Murawaki Y, Shiota G. Retinoic acid receptor alpha dominant negative form causes steatohepatitis and liver tumors in transgenic mice. Hepatology. 2004;40(2):366-75. 